\title{
Fibril elongation mechanisms of HET-s prion-forming domain: Topological evidence for growth polarity
}

\author{
Marco Baiesi, Flavio Seno, and Antonio Trovatd $\circledast$ \\ Department of Physics, Padua University - CNISM, Padova Unit \\ Via Marzolo 8, I-35131 Padova, Italy
}

\begin{abstract}
The prion-forming C-terminal domain of the fungal prion HET-s forms infectious amyloid fibrils at physiological $\mathrm{pH}$. The conformational switch from the non-prion soluble form to the prion fibrillar form is believed to have a functional role, since HET-s in its prion form participates in a recognition process of different fungal strains. Based on the knowledge of the high-resolution structure of HET$\mathrm{s}(218-289)$ (the prion forming-domain) in its fibrillar form, we here present a numerical simulation of the fibril growth process which emphasizes the role of the topological properties of the fibrillar structure. An accurate thermodynamic analysis of the way an intervening HET-s chain is recruited to the tip of the growing fibril suggests that elongation proceeds through a dock and lock mechanism. First, the chain docks onto the fibril by forming the longest $\beta$-strands. Then, the re-arrangement in the fibrillar form of all the rest of molecule takes place. Interestingly, we predict also that one side of the HET-s fibril is more suitable for substaining its growth with respect to the other. The resulting strong polarity of fibril growth is a consequence of the complex topology of HET-s fibrillar structure, since the central loop of the intervening chain plays a crucially different role in favouring or not the attachment of the C-terminus tail to the fibril, depending on the growth side.
\end{abstract}

Keywords: Protein aggregation; Dock and lock mechanism; Coarse-grained models; Monte-Carlo simulations; Topology-based models;

\section{INTRODUCTION}

Prions are self-propagating, usually amyloid-like protein aggregates [1] that are responsible for transmissible diseases. Examples of prion diseases are scrapie in sheep [2], bovine spongiform encephalopathy[3] (BSE) in cattle and new variant Creutzfeldt-Jakob disease [4] in man. In contrast to these disease-related cases, several proteins displaying prion properties are well known in yeast and fungi [5]. The HET-s prion protein produced by the filamentous fungus Podospora anserina is thought to be involved in a specific function. The switching on of its prion form triggers the programmed-cell-death phenomenon named "heterokaryon incompatibility" [6, 7] which can prevent different forms of parasitism, by inducing the death of the heterokaryon formed by cell fusion of different fungal strains. The proteinase K-resistant core of the prion fibrils formed by the C-terminal residues 218289 (PFD:prion forming domain) is unstructured in solution and forms infectious fibrils in vitro [8]. Earlier work showed that HET-s PFD fibrils consist of four $\beta$-strands forming two windings of a $\beta$-solenoid 9$]$ however without giving further information about the details of the intraand inter-molecular $\beta$-sheet architecture. Recently, the structure of HET-s PFD has been determined [10] on the basis of NMR-derived intra and intermolecular distance restraints 11, 12]. This is the only atomic-resolution structural model of an infectious fibrillar state reported to date. On the basis of 134 intramolecular and intermolecular experimental distance restraints, HET-s PFD

\footnotetext{
* Author to whom correspondence should be addressed.

Phone number: +390498277159. Fax number: +390498277102.

Electronic address: trovato@pd.infn.it
}

forms a left-handed $\beta$-solenoid, with each molecule forming two helical windings, a compact hydrophobic core, at least 23 hydrogen bonds, three salt bridges and two asparagine ladders (see Fig. 1a and 1b). The model is supported by electronic diffraction and scanning transmission electronic microscopy [10].

Despite the tremendous economical and social relevance of diseases related to prion infections and protein aggregation and the enormous quantity of research devoted to their study, the mechanisms that rule and trigger fibril formation and elongation remain mostly elusive: the nature of protein aggregation, its limited structural order, its insolubility in water and its involvement with the cell membrane render its experimental study extremely difficult. For these reasons relevant breakthroughs in understanding the principles of amyloid formation and fibril growth might come from numerical simulations.

Recent advances in hardware and methodology have allowed for realistic atomic resolution molecular dynamics (MD) simulations with physics-based potentials of small fibrils consisting of a few monomer units that make possible to span time scales of hundreds of nanoseconds 13 19] and even of $\mu s$ for smaller systems [20 22]. These results were mainly obtained by studying the amyloid $\beta$ peptide $(\mathrm{A} \beta)$, related to the Alzheimer's disease [23], or its mutants and fragments. In this context, experimental observations had first originated the proposal that incoming $\mathrm{A} \beta$ monomers associate to the elongating fibril through a two stages "dock and lock" kinetic mechanism 24. In the first stage, an unstructured monomer docks onto the fibril while maintaining some degree of conformational freedom. In the second stage, the monomer locks into the final fibril state. Such a mechanism was later confirmed and refined, also in the context of $\mathrm{A} \beta$ - 
peptide oligomerization, by other experimental and simulation studies [18, 25, 26]. The dock and lock mechanism was further employed, for the $\mathrm{A} \beta$-peptide, to describe fibril elongation within a thermodynamic context, by means of all-atom MD simulations 27]. A continuous docking stage was observed to occur over a wide temperature range without free energy barriers or intermediates, whereas the locking stage at lower temperatures was characterized by many competing free energy minima in a rugged landscape.

In the case of the fibrillar HET-s PFD protein, it was possible with MD atomistic simulation to probe the stability of the NMR structure on a 10 ns timescale and to predict the behaviour of the salt bridge network [28]. On the other hand, typical elongation times for amyloid fibrils formed by HET-s PFD are of the order of hours 29], so that coarse grained approaches, in which protein chains and amino-acid interactions are modeled in a simplified way, are mandatory to investigate such longer time scales. Indeed, despite the difficulty of finding reliable energy functions, these approaches has been successfully used in outlining general aspects of the full phase diagram of a generic aggregating polypeptide system [30 32], to emphasize the importance of the contribution of hydrophobic interactions and hydrogen bonding in the aggregation process of the $\mathrm{A} \beta$ peptide peptide 33 38] and even to study the mechanisms of monomer addition for the $\mathrm{A} \beta$ peptide and some of its mutants 39 [1] .

In this paper, in order to describe the fibril elongation mechanisms of a relatively long protein domain such as HET-s PFD, we prefer to employ a still different coarsegrained approach since in this case there is the unique advantage of knowing from experiments the fibril structure. At a general level, our strategy falls in the class of approaches used in protein folding that builds on the importance of the native state topology in steering the folding process 42]. In its simplest example, the formation of contacts is favoured only for pairs of amino acids that are found interacting in the native state, but non-native sequence dependent interactions could be introduced as well. Despite the semplicity of this scheme, in the past few years, an increasing number of experimental and theoretical studies have confirmed the utility of the Go-like approach in the characterization of various aspect of protein folding and binding processes [43 50].

The study of protein aggregation has been already tackled by using Go-like models [51, [52], but due to the absence of experimental information on the fibrillar structure, hypothetical aggregate conformations had to be introduced to build the Go-energy function driving aggregation. Moreover, topologically based models, with reduced effect of non-local interactions, correspond to funneled energy landscapes and therefore their application should be limited to situations in which proteins are evolutionarily designed to follow the principle of minimal frustration [53], which results in a faster search through the many possible alternatives. In general, a funneled energy landscape is not expected in the case of non-functional fibril formation. Therefore, the accurate knowledge of its structure, the complex intra-chain topology, and the plausible involvement in a functional process makes HET-s PFD a suitable and, at the moment, unique candidate to exploit successfully Go-like models for studying amyloid formation and fibril elongation mechanisms.

Within this approach, implemented through a MonteCarlo procedure combined with replica exchange methods, we analyze the full thermodynamic properties of the fibril elongation process, e.g. of the association of a free chain to the already formed fibrillar structure of HET-s PFD under different concentration conditions. The behaviour of both energies and heat capacities shows that the association process becomes more cooperative for concentrations in the range $(\sim 10 \mu \mathrm{M})$ of standard invitro experiment [54]. A careful study of the association process shows that fibril elongation is triggered by the docking of the free chain onto the fibril in a concentration dependent mechanism that involves the formation of both inter- and intra-chain hydrogen bonds stabilizing the longest $\beta$-strands, rapidly followed by the assembly of the full domain. This behaviour is similar to the "dock and lock" mechanism proposed for the amyloid A- $\beta$ fibril formation [24, 27].

Another interesting aspect emerges clearly: elongation proceeds differently according to which side of the fibril (see Fig 1a and 1b) is used as the growing end. Elongation from one side is clearly favoured with respect to the other side, implying a strong polarity in the growth of HET-s PFD fibrils, which may even lead to unidirectional elongation. Polarity in fibril growth is a feature already discussed in the literature for other amyloidforming proteins, in both experiments [55, 56] and numerical simulations [27, 57]. Our data suggest that growth polarity can be explained for HET-s PFD on the basis of the complex topological properties of its fibrillar structure. A key role is played by the behaviour of one long loop connecting two $\beta$-strands in consecutive rungs of the fibrillar structure. Depending on the elongation side, this loop may help or not the winding of the C-terminal part of the attaching chain into the fibrillar form. Since it is known that the prion loses its infectivity upon partial deletion of that loop [9], we argue that this topological mechanism may be important for functional fibril growth.

\section{METHODS}

\section{A. Protein chain representation and energy function}

Chains A and B were selected from the NMR structure of HET-s PFD (PDB code: 2rnm), where chain B stays on top of chain A along the fibril. We keep one chain frozen whereas the other one is free. In the top elongation mode, chain B is free and chain A is frozen, whereas in the bottom elongation mode, chain $\mathrm{A}$ is free 
and chain B is frozen. The free chain is allowed to move in a hemisphere of radius $L$ defined by $x^{2}+y^{2}+z^{2}<L^{2}$, $0<z<L(-L<z<0)$ for top (bottom) elongation. The frozen chain is placed with center-of-mass (i.e. average $\mathrm{C}^{\alpha}$ ) coordinates $x_{c m}=y_{c m}=0$ (i.e. on the hemisphere axis) and $z_{c m}=+5.60 \AA\left(z_{c m}=-2.35 \AA\right)$ for top (bottom) elongation, rotated in such a way that the fibril axis is parallel to the hemisphere axis. The centerof-mass position along the hemisphere axis is chosen in order to expose only one 'sticky' end of the frozen chain to the free chain, by placing the other end roughly on the hemisphere base $z=0$. This allows a smaller computational effort, at the expense of prohibiting conformations that we do not expect to affect in a relevant way the binding of the free chain on the exposed end of the full fibril, here represented by the frozen chain. The portion of HET chain that we simulate goes from position 217 to position 289, which includes 73 aminoacids (e.g. in the simulation we include also the MET aminoacids at position 217 used to obtain the NMR structure). We thus simulate a system with 146 aminoacids.

In order to perform extensive simulations, we use a coarse-grained representation of the protein chain coupled with an energy function based on the knowledge of the PDB fibril structure. In the spirit of Go-like approaches widely used for globular proteins [42], the energy function is built in such a way to have its minimum for the PDB structure. Inspired by Ref. [58], each aminoacid is represented by an effective spherical atom located at the position of the corresponding $\mathrm{C}^{\alpha}$ atom. The virtual bond angle associated with three consecutive $C^{\alpha}$ atoms is constrained between $82^{\circ}$ and $148^{\circ}$. Virtual bond lengths are kept constant and equal to the native values from the PDB file. To implement steric constraints we require that no two non-adjacent $C_{\alpha}$ atoms are allowed to be at a distance closer than $3.9 \AA$. We assign an energy -1 to each hydrogen bond that can be formed between two residues that form it in the PDB fibril structure, and we disregard any other attractive interaction (i.e. hydrogen bonds cannot be formed by two residues that do not form it in the PDB fibril structure and we do not consider any other type of pairwise interaction except for the excluded volume constraints). Only $\beta$-sheet stabilizing hydrogen bonds can therefore be formed in our simulation, and in order to identify them within a $C^{\alpha}$-representation, we use the geometrical rules for nonlocal hydrogen bonds introduced in Ref. [58]. In order to recognize the hydrogen bonds present in the PDB fibril structures the lower bound on the scalar product between binormal and connecting vectors was decreased to 0.88 (0.94 in the original formulation, see Table 1 and Fig. 1 in Ref. [58] for the precise listing of all hydrogen bond rules and the definition of binormal and connecting vectors).

In this way, we find in the PDB fibril structure (see Fig. 2 and Fig. 3$)$ two long parallel $\beta$-strands ( $\beta_{1}$ and $\beta_{3}$ ), connected by 9 hydrogen bonds and four shorter strands: $\beta_{2 a}$ parallel to $\beta_{4 a}$ (linked together by 4 hydro- gen bonds) and $\beta_{2 b}$ parallel to $\beta_{4 b}$ (2 hydrogen bonds). Those strands alternate within the fibrillar structure in pairs which form hydrogen bonds within the same chain (intra-chain bonds) and pairs which form hydrogen bonds between neighbouring chains (inter-chain bonds). Each strand in the core of the fibrillar structure forms intrachain bonds on one side and inter-chain bonds on the other side. In the "top" side of the fibril (see Fig. 2 and Fig. 3), the exposed strands in the 'sticky' end are $\beta_{3}, \beta_{4 a} \beta_{4 b}$, whereas in the "bottom" side the exposed strands are $\beta_{1}, \beta_{2 a} \beta_{2 b}$.

Since we keep fixed one chain, the ground state has energy -30 (15 intra-chain hydrogen bonds plus 15 interchain hydrogen bonds - we are not counting the intrachain bonds of the frozen chain). Hence, energy can range from 0 (unbound chains) to -30 (fully bound chains in the fibrillar state). In order to fix a realistic temperature scale, the effective value of hydrogen bond energy in our Go-like energy function was given the value $3.5 \mathrm{Kcal}$. $\mathrm{mol}^{-1}$, so that the unit temperature of our simulations corresponds to $1760 \mathrm{~K}$.

We have simulated the elongation of the fibril from both sides. For each side we simulated chains confined in a hemisphere centred in the origin and of radius $L_{1}=$ $5 \mathrm{~nm}, L_{2}=10 \mathrm{~nm}$, and $L_{3}=30 \mathrm{~nm}$ corresponding to concentrations $c_{1}=6.4 \mathrm{mM}, c_{2}=0.8 \mathrm{mM}$ and $c_{3}=$ $30 \mu \mathrm{M}$. The latter value is close to typical concentrations used in vitro aggregation experiment 29, 54].

\section{B. Monte-Carlo simulation}

Fibril elongation is simulated by means of a Monte Carlo procedure. Multiple Markov processes [59], each replicating the same system of a fixed chain and a free chain attaching to it described above, are run in parallel at different temperatures, with possible swaps of replicas, in order to sample efficiently the conformational space from high $\left(T=210{ }^{\circ} \mathrm{C}\right)$ to low temperatures $\left(T=10^{\circ} \mathrm{C}\right)$. Within the same replica, conformations are evolved using trial moves, which either pivot a part of the chain from a randomly chosen residue to its end, or rotate a chain portion between two residues along the direction joining them. In the latter case the two residues are either chosen randomly or chosen to be next-nearest neighbours along the chain. Trial moves are accepted or rejected according to the Metropolis test [60]. We use 20 different replicas, chosing their temperatures to sample more accurately the transition region $\left(40{ }^{\circ} \mathrm{C}\right.$ $<T<100{ }^{\circ} \mathrm{C}$ ) and to provide reasonable overlap of energy histograms between neighbouring pairs. Roughly $2 \cdot N$ Monte Carlo steps $(N=73$ is the number of residues of the free chain) are performed independently for each replica before one replica swap is attempted among a randomly chosen neighbouring pair. Overall, roughly $10^{7}$ replica swaps are attempted for each simulation and the acceptance rate of replica swaps is in all cases above $60 \%$. The convergence to the equilibrium regime is assessed by 
looking at the evolution of system energy as a function of Monte Carlo steps. In order to compute equilibrium thermodynamic averages, the simulation portion corresponding to the initial $N_{e q}$ swaps is discarded from the collected data, with $N_{e q}$ ranging from $1.5 \cdot 10^{6}$ to $5 \cdot 10^{6}$, depending on concentration. Data from all temperatures are elaborated with the multiple-histogram method [61]. In Fig. 4 some snapshots of the simulations are represented.

\section{RESULTS}

\section{A. Cooperativity of the elongation process}

To characterize the thermodynamic properties of the HET-s fibril growth process we study the behaviour of the energy and of the heat capacity of the system as a function of the temperature for three different protein concentrations (see Fig. 5) and by considering elongation both from the top and from the bottom side (see Methods). The peaks in the heat capacity curve signal the occurrence of large conformational rearrangements (that strictly speaking would become transitions only in the thermodynamic limit of the number of system component going to infinite) related to the process of the deposition of the free HET-s PFD chain to the one that is kept fixed to represent the sticky end of the already formed fibril.

As expected, the first association temperature, related to the high temperature heat capacity peak, decreases when lowering the concentration. It reaches values close to room temperature for the concentration $c_{3}=30 \mu \mathrm{M}$. Interestingly, the cooperativity of the transition increases for lower concentrations: this is signalled by a more sigmoidal behaviour of the energy profiles, by the sharpening of heat capacity peaks and by the almost complete merging in one single narrow peak of the otherwise complex peak structure. Despite at a first glance the growth processes from the two different sides look similar, one can notice that at low concentration a residual peak remains at low temperature for the top elongation case.

\section{B. Stability of hydrogen bond formation}

To elucidate further these behaviours and to understand the nature of the conformational rearrangements related to heat capacity peaks we computed the formation probability of hydrogen bonds for the different strands as a function of temperature (shown in Fig. 6). We define the stabilization temperature of a group of hydrogen bonds to correspond to their average formation probability at thermodynamic equilibrium being equal to 0.5. We compute stabilization temperatures for 6 possible groups of hydrogen bonds, corresponding to the different strand pairings shown as separate white/black bands in Fig. 6. The resulting stabilization temperatures are summarized in Table \.

We will use the term 'fibrillar' for inter-chain hydrogen bonds. As an example, strand $\beta_{1}$ of the mobile chain couples with strand $\beta_{3}$ of the fixed chain, in the case of elongation from the top side; whereas strand $\beta_{3}$ of the mobile chain couples with strand $\beta_{1}$ of the fixed chain in the case of elongation from the bottom side, and similarly for other strand pairs (see Fig. 6 caption for a detailed explanation of how intra-chain and fibrillar hydrogen bonds are represented in the figure).

For all concentrations and for both elongation sides, the first hydrogen bonds which become stable are the inter-chain ones formed between the long strands $\beta_{1}$ and $\beta_{3}$, at a temperature $T_{f 13}$ varying from $115^{\circ} \mathrm{C}$ for top elongation at concentration $c_{1}$, to $65^{\circ} \mathrm{C}$ for bottom elongation at $c_{3}$ (see Table I). The stabilization temperature $T_{f 13}$ of fibrillar hydrogen bonds between the long strands is higher for higher concentrations and, at the same concentration, for the case of top side elongation.

This first stabilization process is followed at a lower temperature $T_{i 13}$ (varying from $85^{\circ} \mathrm{C}$ for top elongation at concentration $c_{1}$, to $63^{\circ} \mathrm{C}$ for bottom elongation at $c_{3}$, see Table I) by the stabilization of intra-chain hydrogen bonds formed between the two long strands, $\beta_{1}$ and $\beta_{3}$, of the mobile chain. This second stabilization temperature is again higher for the top elongation case, at the same concentration, and increases with concentration.

With a further decrease of the temperature the fibrillar hydrogen bonds formed between the short strands ( $\beta_{2 a}$ with $\beta_{4 a}$ and $\beta_{2 b}$ with $\beta_{4 b}$ ) become stable. The stabilization temperature $T_{f 24 a}$ for inter-chain strand pair $\beta_{2 a}$ and $\beta_{4 a}$ does not depend on concentration but only on the elongation side, being higher, $T_{f 24 a} \simeq 63{ }^{\circ} \mathrm{C}$, for top elongation with respect to $T_{f 24 a} \simeq 50{ }^{\circ} \mathrm{C}$ for bottom elongation. The stabilization temperature $T_{f 24 b}$ for inter-chain strand pair $\beta_{2 b}$ and $\beta_{4 b}$ is roughly the same, $T_{f 24 b} \simeq 42 \div 43^{\circ} \mathrm{C}$, in all cases.

The last step involves the stabilization of the intrachain hydrogen bonds between the short strands and do not depend as well on concentration. At this stage the more significant difference between the two elongation sides emerges. For top side elongation, the two short strand pairs are stabilized roughly together at a temperature much lower with respect to the previous step $\left(T_{i 24 a} \simeq 10{ }^{\circ} \mathrm{C}\right.$ corresponding to the small peak in the heat capacity curve and $T_{i 24 b} \simeq-2{ }^{\circ} \mathrm{C}$ ). For bottom side elongation, the stabilization of the two short intra-chain strand pairs takes place at quite different temperatures: hydrogen bonds between strands $\beta_{2 a}$ and $\beta_{4 a}$ are stabilized at a even slightly higher temperature than their inter-chain counterpart $\left(T_{i 24 a} \simeq 44{ }^{\circ} \mathrm{C}\right)$, whereas the intra-chain strand pair $\beta_{2 b}$ and $\beta_{4 b}$ is stabilized at a much lower temperature $\left(T_{i 24 b} \simeq 6{ }^{\circ} \mathrm{C}\right)$. 


\section{C. 'Dock and lock' mechanism}

Upon assuming that the order in hydrogen bond stabilization mirrors a similar order in the kinetics of the elongation process, we can extract a general message from these results by stating that the attaching of a mobile chain onto the elongating fibril is triggered by the formation of the inter-chain hydrogen bonds of the longest strand $\left(\beta_{1}\right.$ or $\left.\beta_{3}\right)$ followed by a first partial folding of the chain through the formation of the long intra-chain strand pair (between $\beta_{1}$ and $\beta_{3}$ ) and by the successive formation of all other inter- and intra-chain hydrogen bonds. The mechanism of addition of a soluble unstructured monomer to a preformed ordered amyloid fibril is a complex process: the deposition involves an association of the unstructured monomer to the fibril surface (docking) followed by a conformational rearrangement leading to the incorporation onto the underlying fibril lattice (locking) 18, 24 26.

We identify the docking stage with the formation of both inter- and intra-chain hydrogen bonds between the long strands $\beta_{1}$ and $\beta_{3}$, as in both cases the stabilization temperatures $T_{f 13}$ and $T_{i 13}$ depends on concentration (see Table I). One indeed expects that in a denser regime it is easier for the mobile chain to dock onto the fibril end, while the locking into the $\beta$-helix structure necessary for fibril propagation should not be affected by concentration changes. Therefore, the locking stage involves the formation of both inter- and intra-chain hydrogen bonds between the remaining shorter strands, since we observe that their stabilization temperatures do not depend on concentration. The dependence of the intrachain hydrogen bond stabilization temperature $T_{i 13}$ upon concentration is non-trivial and is triggered by the strong concentration dependence of the formation probability of the fibrillar hydrogen bonds between the long strands. The higher the concentration, the more probable the fibrillar bonds to be formed. Consequently, the more easily the intra-chain bonds are stabilized.

From the data shown in Fig. 6 and in Table I another general feature can be picked out: the temperature range in which the docking stage and thus the full elongation process take place decreases at lower concentrations. This is consistent with the increment of cooperativity as it appears from thermodynamic quantities (e.g. sharpness of heat capacity peaks in Fig. 5) when concentration diminishes. The most cooperative behavior, as shown by the presence of a single sharp peak in the heat capacity curve, is obtained at concentration $c_{3}$ in the case of bottom side elongation, whereas a second peak is clearly visible at low temperature for top side elongation at the same concentration. The above analysis of Fig. 6 data reveals the crucial role of the formation of intra-chain hydrogen bonds between strands $\beta_{2 a}$ and $\beta_{4 a}$ in this respect. The stabilization temperature $T_{i 24 a}$ of these hydrogen bonds constitutes the most relevant difference between the two elongation modes in the first place. Moreover, it does correspond closely in both cases to significant features in the heat capacity curve, namely the small peak at $T_{i 24 a} \simeq 10{ }^{\circ} \mathrm{C}$ for top elongation and the small shoulder in the main peak at $T_{i 24 a} \simeq 44{ }^{\circ} \mathrm{C}$ for bottom elongation.

\section{Competition between intra-chain and inter-chain hydrogen bonds}

In order to gain a further understanding of the role played by intra-chain $\beta_{2 a}-\beta_{4 a}$ hydrogen bonds we computed for different temperatures the equilibrium occupation probabilities of macroscopic conformational states, which are defined according to the number of formed intra- or inter-chain hydrogen bonds. In Fig. 7 and Fig. 8 the results are shown for concentration $c_{3}$ and for the two different growth modes. Occupation probabilities are shown in logarithmic scale, so that the resulting data could be interpreted as (the opposite of) effective free energies or mean force potentials. At high temperature, the bottom left corner is mostly populated, corresponding to conformations with very few or none intra- and inter-chain hydrogen bonds formed, typical of a mobile chain not yet attached to the fibril end. On the other hand, at very low temperature, the opposite top right corner is populated, describing structures with almost all the hydrogen bonds formed that correspond to mobile chains found already completely associated to the fibril end with a significant probability.

Consistently with the previous analysis, based on Fig. 6 data, the elongation process is complex, taking place in several stages. Macrostates with only intra-chain hydrogen bonds are found to be populated to some extent at high temperatures, hinting to the possibility of a prestructuring of the mobile chain before docking to the fibril tip, yet the pathway more significantly visited involves the population of first the 9 fibrillar hydrogen bonds between strand $\beta_{1}$ and $\beta_{3}$ and then the analogous intrachain bonds (see $T=70{ }^{\circ} \mathrm{C}$ in Fig. 7 and Fig. 8).

After this first stage, that we already identified with docking, two different scenarios emerge again clearly, depending on the growth mode. The overall process is visibly more cooperative for bottom side elongation (Fig. 8) with respect to top side elongation (Fig. 7), since the spreading of significantly visited macrostates is restricted to a narrower temperature range in the former case. Moreover, around room temperature $\left(T=20-30{ }^{\circ} \mathrm{C}\right)$, the most populated state for bottom side elongation has 15 fibrillar and 13 intra-chain hydrogen bonds formed corresponding to a chain almost completely attached to the fibril end (only strand $\beta_{2 b}$ is left wiggling a bit). Instead, the most populated state for top side elongation has all 15 inter- but only 9 intra-chain hydrogen bonds locked into the fibrillar conformation, signalling again that the stability of intra-chain $\beta_{2 a}-\beta_{4 a}$ hydrogen bonds is strongly weakened with respect to bottom side elongation.

In order to appreciate more easily the variations 
with temperature in the population of the different macrostates, we computed unidimensional free energy profiles as a function of the number of either intra- or inter-chain hydrogen bonds. The results are shown in Fig. 9 for concentration $c_{3}$ and for the two different growth modes.

Fig. 9 pictures confirm the multistage nature of the association process with different macrostates that become the global free energy minimum at different temperatures. The free energy profiles as a function of the number of fibrillar hydrogen bonds are similar in both elongation modes. The main difference is the value of the temperature below which the free state (none fibrillar hydrogen bond is formed) is not the free energy minimum anymore: $70{ }^{\circ} \mathrm{C}$ for top elongation and $60{ }^{\circ} \mathrm{C}$ for bottom elongation, consistently with Table —

The free energy of the free state and the free energy barrier that separates it from the competing minimum with 9 fibrillar bonds (the ones formed between the $\beta$ strands $\beta_{1}$ and $\beta_{3}$ that are the first to be stabilized in the association process) do not basically depend on temperature. This is a signature of their entropic origin (free energies in fig. 9 are plotted in $R T$ units), as they are both dominated by the roto-translational entropy of the free chain.

On the other hand, the free energy profiles as a function of the number of intra-chain hydrogen bonds display a relevant difference between the two elongation modes, consistently with previous observations. For top elongation, the free energy minimum at $T=20{ }^{\circ} \mathrm{C}$ is the macrostate with only 9 intra-chain hydrogen bonds (i.e. the $\beta$-strands $\beta_{2 a}$ and $\beta_{4 a}$ are not yet paired), whereas for bottom elongation the free energy minimum at $T=20^{\circ} \mathrm{C}$ is the macrostate with 13 intra-chain hydrogen bonds (i.e. the $\beta$-strands $\beta_{2 a}$ and $\beta_{4 a}$ are already paired).

Interestingly, the above difference is due to the macrostate with 9 intra-chain hydrogen bonds being entropically stabilized for top elongation with respect to bottom elongation. Indeed, the free energies for $10 \div 15$ intra-chain hydrogen bonds do not differ in the two elongation modes. Moreover, the free energy difference between the two elongation modes for 9 intra-chain hydrogen bonds has to be entropic, since the energy of that macrostate is the same in both cases, being given by the 9 intra-chain plus the 15 inter-chain hydrogen bonds (the latter is the global free energy minimum at $T=20{ }^{\circ} \mathrm{C}$ for both elongation modes).

\section{E. Topological origin of fibril growth polarity}

Our analysis clearly established that fibril growth exhibits a deeply different thermodynamic behaviour depending on the side from which elongation proceeds: at room temperature only bottom side elongation is thermodynamically stable. What is the physical reason for the existence of such a strong growth polarity? Since our simulation study is based only on the knowledge of the fibril structure and not on the specificity of the aminoacid sequence, we can expect that growth polarity is a consequence of the topological properties of the structure.

There is indeed a deep topological difference between the deposition mechanisms of HET-s PFD on the two different sides of the fibril. In the first docking stage, common to both elongation modes, the formation of both inter- and intra-chain hydrogen bonds between $\beta_{1}$ and $\beta_{3}$ implies the positioning of the latter strands into a conformation already compatible with the final fibrillar structure. The remaining strands, then, yet to be positioned, acquire distinct topological roles, since $\beta_{2 a}$ and $\beta_{2 b}$ are in a loop between the two chain portions already pinned down in the fibrillar structure, whereas $\beta_{4 a}$ and $\beta_{4 b}$ are in the $\mathrm{C}$-terminal tail of the chain. One can then predict that, for entropic reasons, the former pair can be accomodated into the final fibrillar structure more easily than the latter pair. Nevertheless, different elongation modes may change this hierarchy.

When elongation proceeds from the top side, the attaching chain wraps up onto the fibril tip starting with its N-terminal part (see Fig. 10). The 'loop' strands $\beta_{2 a}$ and $\beta_{2 b}$ (fibrillar) need to be positioned before the 'tail' ones $\beta_{4 a}$ and $\beta_{4 b}$ (intra-chain), consistently with the topological order suggested above. Indeed we do observe this hierarchy for top side elongation; even at low temperature intra-chain hydrogen bonds between the short strand pairs are not yet stable.

When elongation proceeds from the bottom side, the attaching chain wraps up onto the fibril tip starting with the C-terminal part (see Fig. 10). The 'tail' strands $\beta_{4 a}$ and $\beta_{4 b}$ (fibrillar) need now to be positioned before the 'loop' ones $\beta_{2 a}$ and $\beta_{2 b}$ (intra-chain). Elongation order takes over the topological order so that the difficult positioning of 'tail' strands is assisted by the easier positioning of 'loop' strands and both are stabilized at room temperature (with the partial exception of the shortest strand $\left.\beta_{2 b}\right)$.

\section{DISCUSSION}

In this work we have used Monte-Carlo simulations of a coarse-grained representation of HET-s PFD domain in order to get information about the elongation of the corresponding amyloid fibril by attaching of a mobile chain to a pre-fixed fibrillar structure. Our approach, based on the knowledge of the fibrillar structure, relies on the currently well accepted assumption that protein topology plays a pivotal factor in determining unimolecular folding and protein assembly. At variance with other Go-like studies [51, 52], based on a hypothetical structure, the reliability of our study is justified by the knowledge of a high-resolution NMR structure of a plausibly functional amyloid fibril.

There are two main results of our thermodynamic study. First, we observe that fibril elongation is driven 
by the formation of inter-chain and intra-chain hydrogen bonds between the long strands $\beta_{1}$ and $\beta_{3}$, followed by the positioning of the rest of the attaching chain onto the growing fibril. This mechanism is known as dock and lock mechanism [18, 24]. We identify the docking stage as that part of the association process whose onset temperature displays a concentration dependance. A similar feature, i.e. the docking temperature range varies with concentration, was previously found in a thermodynamic study of $\mathrm{A} \beta$-peptide fibril growth [27]. Within our Golike approach, we find a complex multistage association process, where both the docking and the locking stages proceed in several steps in a free energy landscape characterized by several different minima separated by barriers. In the case of $\mathrm{A} \beta$-peptide fibril elongation, it was found on the contrary that docking is continuous and proceeds without the presence of intermediate or free energy barriers [27]. Our finding of a multistage docking behaviour might be due to the non-trivial intra-chain topology of HET-s PFD monomer, lacking in the $\mathrm{A} \beta$-peptide case. Alternatively, it might be an artifact caused by our neglecting of non-native interactions.

Secondly, we predict that one side of the structure is more suitable to substain the growth of the fibril.

The predicted fibril growth polarity can be rationalized by analyzing elongation topological properties, which turn out to be intrinsically different from the two fibril sides. The argument is based on the complex tertiary structure of the monomeric unit within HET-s fibril, resulting into alternating intra- and inter-chain pairs of $\beta$-strands. After the first hydrogen bonds have been formed in the initial docking onto the growing fibril, the portion of the attaching chain which is going to acquire $\beta$-structure in the following locking stage, is partitioned into a 'loop' and a 'tail' part (see Fig. 10). As a consequence, the entropy loss of the two parts upon locking is different, implying a 'topological' hierarchy. The latter may or may not be affected by the 'winding' hierarchy dictated by the choice of the growth side, thus resulting in a strong growth polarity. Bottom side elongation is more sustainable because the 'loop' part may assist the 'tail' part to lock. The entropic origin of the growth polarity observed in our results is further confirmed by the free energy profiles shown in Fig. 9. As discussed in section IIID, the macrostate populated after docking and before locking (with 15 inter-chain and 9 intra-chain hydrogen bonds) is entropically stabilized in the top elongation mode with respect to bottom elongation.

Being based on a topological argument, we believe our prediction to be robust against both variations of the details in the implementation of our Go-like approach (changing the coarse-graining of protein chain representation, employing in the energy function general pairwise contacts and not only hydrogen bonds, using different hydrogen bond rules) and relaxation of other simplifying assumptions that we made, namely the fibril tip is represented by just one chain and kept completely frozen. This latter point is further motivated by the experimen- tal observation [29] that the HET-s fibril accomodates incoming prion monomers without a substantial disorganization of its structure. This behaviour is quite different from those of $\mathrm{A} \beta$ fibrils where it was experimentally observed [62] an entropy gain in the elongation reaction which was related to an unfolding of the organized fibril end to accomodate the addition of the incoming monomers.

We thus suggest our prediction may be accessible to experimental validation, for instance using the single fiber growth assay employed in [56] for SUP35 yeast prion, based on two variants of the prion domain that can be differentially labelled and distinguished by atomic force microscopy.

The same topological argument cannot be used for simpler amyloid structure such as the solid state NMR-model suggested for the Alzheimer's $A \beta$-peptide [63], in the absence of intra-chain hydrogen bonds. In fact, experimental evidence shows bidirectional fibril growth with no clear signs of growth polarity 55. Interestingly, the presence of asymmetrical topological properties of fibril ends was indeed suggested for the $A \beta$-peptide [27, 57], depending on the different possible quaternary arrangements of the fibril 64. On the other hand, a clear evidence of growth polarity was demonstrated for SUP35 fibrils [56], a yeast prion protein believed to have a functional role, similarly to HET-s prion. There is no high-resolution information on the structure of SUP35 fibrils and different conflicting structural models have been recently proposed 65, 66], yet some of the available data suggests the existence of a complex intra-chain structure [67], which could justify the observed growth polarity within a framework similar to the one we propose here for HET-s.

A peculiar feature of HET-s PFD is the presence of a long loop in the fibrillar structure that connects strands $\beta_{2 b}$ and $\beta_{3}$ (aa 246-260), which then contributes to increasing the length of the chain portion partitioned as 'loop' in the topological argument discussed above (in blue in Fig. 10). An interesting question is whether increasing such length favours or disfavours growth polarity according to the mechanism suggested here. One could argue that a longer loop may further decrease the fluctuations available to the 'tail' part, thus enhancing its assisted locking for bottom side elongation. Indeed, it is known that a large deletion in such loop (248-256) led to loss of HET-s function and infectivity [9]. Even though no evidence is known about the possible biological relevance of fibril growth polarity in fungal and yeast prions, it is tempting to speculate that the role of the loop might be to enhance growth polarity as a way to control the elongation process more thoroughly in the context of the propagation of a functional prion. Numerical simulations performed within the same Go-like approach, based on a model structure to be built on the basis of HET-s PFD but with a shorter loop, will shed further light on this hypothesis.

Our coarse-grained approach turned out to be effective in studying structural rearrangements which could not 
be tackled using more detailed protein chain representations. On the other hand, we are neglecting factors which may play an important role in the elongation process, such as the possible presence of oligomeric conformers of HET-s PFD in the non-fibrillar soluble state [29]. In order to capture similar effects, one needs to increase the accuracy of the energy function by introducing aminoacid specificity. This can be done by using coarse-grained potentials which take into account the different ability of each pair of amino-acids in forming hydrogen bonds in $\beta$-strands [68, 69], coupled with similar potentials describing residue pairwise interactions [70] or local conformational biases [71]. Such potentials might be used to modulate the Go-like energy function presented in this work. The use of a more realistic description of the protein chain involving side chain atoms may also cause the amino-acid sequence to affect the elongation process in a side-depending manner by imposing chiral stereochemical constraints. The latter are not present in our $\mathrm{C}^{\alpha}$-based representation, thus reinforcing the topological origin of growth polarity in our results.
Finally, the approach presented here could be also used to study the nucleation process of HET-s PFD, and further extended to study the aggregation of the full HETs protein. The HET-s N-terminal domain in the nonfibrillar soluble form is structured into a globular protein fold whose high-resolution structure has been very recently released [54] (PDB code 2wvn.pdb). Such structure is known to lose partially its order upon ordering and aggregation of HET-s PFD into the unsoluble fibrillar form [2]. A Go-like approach would be especially suited to study the resulting competition between the ordered structures of the two domains in the two different forms [73].

\section{ACKNOWLEDGMENTS}

We thank F. Chiti and R. Riek for enlightening discussions. We acknowledge financial support from University of Padua through Progetto di Ateneo n. CPDA083702.
[1] A. Aguzzi, C. Sigurdson, and M. Heikenwaelder, Annu. Rev. Phatol. 3, 11 (2008).

[2] S. Prusiner, Science 216, 136 (1982).

[3] G. Wells, A. Scott, C. Johnson, R. Gunnig, R. Hancock, M. Jeffrey, M. Dawson, and R. Bradley, Veterinary Record 121, 419 (1987).

[4] R. Will, J. Ironside, M. Zeidler, S. Cousens, K. Estibiero, A. Alperovitch, S. Poser, M. Pocchiari, A. Hofman, and P. Smith, Lancet 347, 921 (1996).

[5] M. Tuite and T. Serio, Nat. Rev. Mol. Cell. Biol. 11, 823 (2010).

[6] A. Lange and B. Meier, Comptes Rendue Chemie 11, 332 (2008).

[7] S. Saupe, Microbiol. Mol. Biol. Rev. 64, 489 (2000).

[8] A. Baulgerie, S. D. Reis, C. Ritter, B. C.-S. S. Chaignepain, V. Forge, K. Bathany, I. Lascu, J. Schmitter, R. Riek, and S. Saupe, EMBO J. 22, 2071 (2003).

[9] C. Ritter, M. Maddelein, A. Simmer, T. Luhrs, M. Ernst, B. Meier, S. Saupe, and R. Riek, Nature 435, 844 (2005).

[10] C. Wasmer, A. Lange, H. V. Melckebe, A. Siemer, R. Riek, and B. Meier, Science 319, 1523 (2008).

[11] F. Castellani, B. van Rossum, A. Diehl, M. Schubert, K. Rehbein, and H. Oschkinat, Nature 420, 98 (2002).

[12] A. Lange, S. Becker, K. Seidel, K. Giller, O. Pongs, and M. Baldus, Angew. Chem. Int. Ed. 44, 2441 (2005).

[13] A. Baumketner and J. Shea, J. Mol. Biol. 366, 275 (2007).

[14] N. Buchete and G. Hummer, Biophys. J. 92, 3032 (2007).

[15] M. Cecchini, R. Curcio, M. Pappalardo, R. Mekl, and A. Caflisch, J. Mol. Bio. 357, 1306 (2006).

[16] A. Huet and B. Derremaux, Biophys. J. 91, 3829 (2006).

[17] B. Ma and R. Nussinov, Curr.Opin. Chem. Biol. 10, 445 (2006).

[18] E. O'Brien, Y. Okamoto, J. Straub, B. Brooks, and D. Thirumalai, Journal of Physical Chemistry B 113, 14421 (2009).

[19] B. Tarus, J. Straub, and D. Thirumalai, J. Am. Chem.
Soc. 128, 16159 (2006).

[20] G. Reddy, J. Straubb, and D. Thirumalai, Proc. Natl. Acad. Sci. USA 106, 11948 (2009).

[21] T. Takeda and D. Klimov, J. Phys. Chem. B 114, 4755 (2010).

[22] G. Wei, A. Jewett, and J. Shea, Phys. Chem. Chem. Phys. 12, 3622 (2010).

[23] J. Hardy and D. Selkoe, Science 297, 353 (2002).

[24] W. Esler, E. Stimson, H. V. J.J. Jennings, J. Ghilardi, J. Lee, P. W. Mantyh, and J. Maggio, Biochemistry 39, 6288 (2000).

[25] M. Cannon, A. Williams, R. Wetzel, and D. Myszka, Anal. Biochem. 328, 67 (2004).

[26] J. Straub and D. Thirumalai, Current Opinion in structural biology 20, 187 (2010).

[27] T. Takeda and D. Klimov, Biophys. J. 96, 442 (2009).

[28] A. Lange, Z. Gattin, H. van Meleckebeke, C. Wasmer, A. Soragni, W. van Gunsteren, and B. Meier, ChemBioChem 10, 1657 (2009).

[29] R. Sabaté, V. Castillo, A. Espargaró, S. Saupe, and S. Ventura, FEBS J. 276, 5053 (2009).

[30] S. Auer and D. Kashiev, Proteins-Structure Function and Bioinformatics 78, 2412 (2010).

[31] S. Auer, A. Trovato, and M. Vendruscolo, Plos Computational Biology 5, e1000458 (2009).

[32] T. Hoang, L. Marsella, A. Trovato, F. Seno, J. Banavar, and A. Maritan, Proc. Natl. Acad. Sci. USA 103, 6883 (2006).

[33] J. Borroguero, B. Urbanc, N. Lazo, S. Buldyrev, D. Teplow, and H. Stanley, Proc. Natl. Acad. Sci. USA 102, 6015 (2005).

[34] G. Favrin, A. Irback, and S. Mohanty, Biophys. J. 87, 3657 (2004).

[35] N. Fawzi, V. Chubukov, L. Clark, S. Brown, and T. HeatGordon, Protein Sci. 14, 993 (2005).

[36] H. Nguyen and C. Hall, J. Am. Chem.Soc. 128, 1890 (2006). 
[37] B. Urbanc, L. Cruz, F. Ding, D. Sammond, S. Khare, S. Buldyrev, H. Stanley, and N. Dokholyan, Biophys. J. 87, 2310 (2004).

[38] G. Wei, N. Mousseau, and P. Derreumaux, Biophys. J. 87, 3648 (2004).

[39] A. Lam, D. B. Teplow, H. Stanley, and B. Urbanc, J. Am. Chem. Soc. 130, 17413 (2008).

[40] A. Rojas, A. Liwo, D. Browne, and H. Scheraga, J. Mol. Biol. 404, 537 (2010).

[41] B. Urbanc, M. Betnel, L. Cruz, G. Bitan, and D. Teplow, J. Am. Chem. Soc. 132, 4266 (2010).

[42] N. Go and H. Scheraga, Macromolecules 9, 535 (1976).

[43] E. Alm and D. Baker, Current Opinion in Structural Biology 9, 189 (1999).

[44] D. Baker, Nature 405, 39 (2000).

[45] C. Clementi, H. Nymeier, and J. Onhucic, J. Mol. Biol. 298, 937 (2000).

[46] I. Hubner, M. Oliveberg, and E. Shakhnovich, Proc. Natl. Acad. Sci. USA 101, 8354 (2004).

[47] A. Lam, J. Borreguero, F. Ding, N. Dokholyan, S. Buldyrev, H. Stanley, and E. Shaknovich, J. Mol. Biol. 373, 1348 (2007).

[48] Y. Levy, S.S.Cho, J. Onuchic, and P. Wolynes, J. Mol. Biol. 346, 1121 (2005).

[49] C. Micheletti, J. Banavar, A. Maritan, and F. Seno, Phys. Rev. Lett. 82, 3372 (1999).

[50] J. Sulkowska, P. Sulkowski, and J. Onuchic, Proc. Natl. Acad. Sci. USA 106, 3119 (2009).

[51] F. Ding, N. Dokholyan, S. Buldyrev, H. Stanley, and E. Shaknovich, J. Biol. Mol. 324, 851 (2002).

[52] H. Jang, C. Hall, and Y. Zhou, Biophys. Journal 86, 31 (2004).

[53] J. Bryngelson and P. Wolynes, Proc. Natl. Acad. Sci. USA 84, 7524 (1987).

[54] J. Greenwald, C. Buthz, C. Ritter, W. Kwiatkowski, S. Choe, M. Maddelein, F. Ness, S. Cescau, A. Soragni, D. Leitz, et al., Molecular Cell 38, 889 (2010).

[55] H. Blackley, G. Sanders, M. Davies, C. Roberts, S. Tendler, and M. Wilkinson, J. Mol. Biol. 298, 833 (2000).

[56] A. DePace and J. Weissman, Nat. Struct. Biol. 9, 389 (2002).

[57] N. Fawzi, Y. Okabe, E.-H.Yap, and T. Head-Gordon, J. Mol. Biol. 365, 535 (2007).

[58] T. Hoang, A. Trovato, F. Seno, J. Banavar, and A. Maritan, Proc. Natl. Acad. Sci. USA 101, 7960 (2004).

[59] M. Tesi, E. vanRensburg, E. Orlandini, and S. Whittington, J. Stat. Phys. 82, 155 (1996).

[60] N. Metropolis, A. Rosenbluth, M. Rosenbluth, A. Teller, and E. Teller, J. Chem. Phys. 21, 87 (1953).

[61] F. Wang and D. Landau, Phys. Rev. Lett. 86, 2050 (2001).

[62] Y. Kusumoto, A. Lomakin, D. Teplow, and G. Benedek, Proc. Natl. Acad. Sci. USA 95, 12277 (1998).

[63] A. Petkova, Y. Ishii, J. Balbach, O. Antzutkin, D. Gordon, S. Meredith, and R. Tycko, Proc. Natl. Acad. Sci. USA 99, 16742 (2002).

[64] A. Petkova, W.-M. Yau, and R. Tycko, Biochem. 45, 498 (2006).

[65] R. Krishnan and S. Lindquist, Nature 447, 453 (2005).

[66] F. Shewmaker, R. Wickner, and R. Tycko, Proc. Natl. Acad. Sci. USA 103, 19754 (2006).

[67] P. Tessier and S. Lindquist, Nat. Struct. Mol. Biol. 16, 598 (2009).
[68] A. Trovato, F. Chiti, A. Maritan, and F. Seno, Plos Computational Biology 2, 1608 (2006).

[69] A. Trovato, F. Seno, and S. Tosatto, Protein Engineering Design and Selection 20, 521 (2007).

[70] S. Miyazawa and R. L. Jernigan, J. Mol. Biol. 256, 623 (1996).

[71] Q. Fang and D. Shortle, Proteins 60, 90 (2005).

[72] C. Wasmer, A. Shutz, A. Loquet, C. Buhtz, J. Greenwald, R. Riek, A. Bockmann, and B. Meier, J. Mol. Biol. 319, 1523 (2009).

[73] M. Zamparo, A. Trovato, and A. Maritan, Phys. Rev. Lett. 105, 108102 (2010). 
TABLE LEGENDS

\begin{tabular}{|l|c|c|c|c|c|c|}
\hline & $T_{f 13}\left({ }^{\circ} \mathrm{C}\right)$ & $T_{i 13}\left({ }^{\circ} \mathrm{C}\right)$ & $T_{f 24 a}\left({ }^{\circ} \mathrm{C}\right)$ & $T_{f 24 b}\left({ }^{\circ} \mathrm{C}\right)$ & $T_{i 24 a}\left({ }^{\circ} \mathrm{C}\right)$ & $T_{i 24 b}\left({ }^{\circ} \mathrm{C}\right)$ \\
\hline \multicolumn{7}{|c|}{ top side elongation } \\
\hline$c_{1}$ & 115 & 85 & 64 & 43 & 12 & -1 \\
$c_{2}$ & 88 & 71 & 63 & 44 & 11 & -2 \\
$c_{3}$ & 75 & 70 & 62 & 43 & 8 & -3 \\
\hline \multicolumn{7}{|c|}{ bottom side elongation } \\
\hline$c_{1}$ & 108 & 80 & 50 & 42 & 45 & 6 \\
$c_{2}$ & 85 & 68 & 47 & 40 & 43 & 7 \\
$c_{3}$ & 65 & 63 & 50 & 41 & 44 & 6 \\
\hline
\end{tabular}

TABLE I. Stabilization temperatures of hydrogen bond groups for the different concentrations and elongation modes used in our simulations. Hydrogen bonds stabilizing a given strand pair are grouped into the same set, resulting into 6 different sets (see Fig. 6 caption for a detailed explanation). Stabilization temperatures for different sets are labeled in such a way that, e.g., $T_{f 13}\left(T_{i 24 a}\right)$ refers to fibrillar (intra-chain) hydrogen bonds formed between strands $\beta_{1}$ and $\beta_{3}\left(\beta_{2 a}\right.$ and $\left.\beta_{4 a}\right)$. 


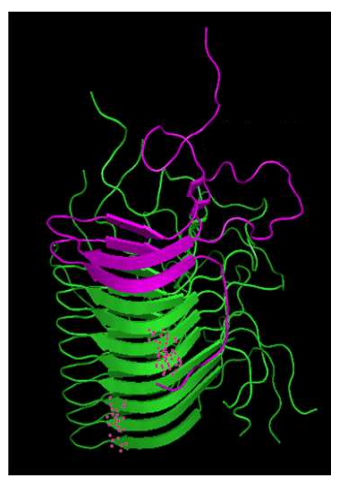

a)

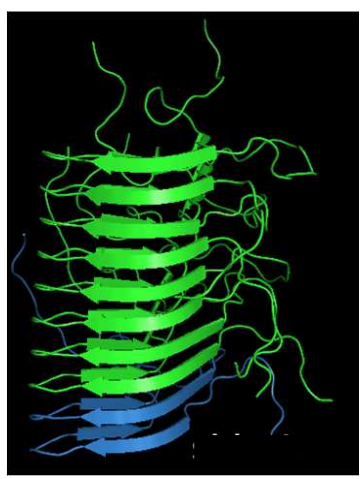

b)

FIG. 1. High resolution experimental structure of HET-s fibril. Side view of the five molecules present in the fibrillar structure of HET-s PFD calculated with NMR restraints (PDB code:2RNM). In a) the monomer on the top of the fibril (chain $\mathrm{E}$ ) is drawn in violet, whereas in b) the bottom monomer (chain A) is drawn in blue
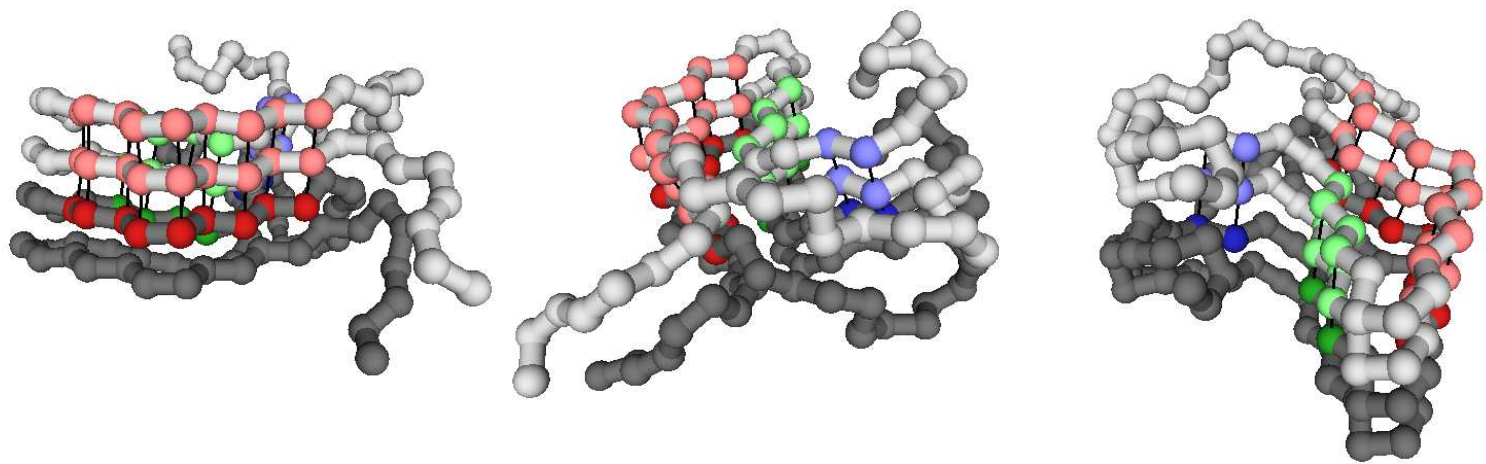

FIG. 2. Coarse-grained represention of HET-s molecules (top side elongation). Different views of the HET-s PFD structure used as the minimum energy structure in our simulations. Chains A and B from the PDB fibril structure are kept. During the simulation of top side elongation, the light chain is mobile while the dark one is frozen and represents the fibril end to which the mobile chain is attaching. The three types of $\beta$-stranded regions are depicted in red $\left(\beta_{1} / \beta_{3}\right)$, green $\left(\beta_{2 a} /\right.$ $\left.\beta_{4 a}\right)$, and blue $\left(\beta_{2 b} / \beta_{4 b}\right)$. The hydrogen bonds used in the Go-like energy functions are represented by dark lines joining the interacting $\mathrm{C}^{\alpha}$ atoms.
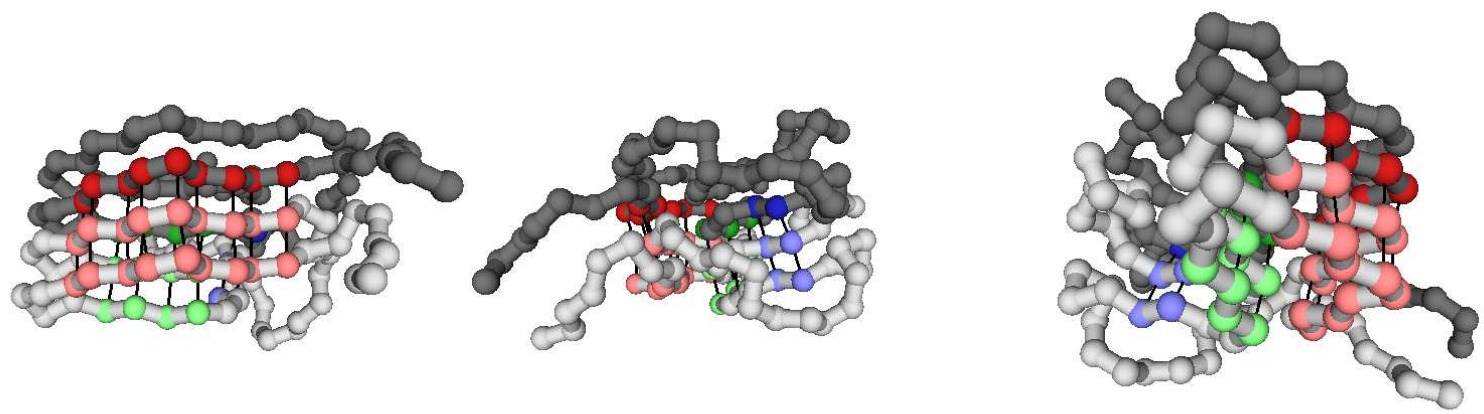

FIG. 3. Coarse-grained represention of HET-s molecules (bottom side elongation). Different views of the HET-s PFD structure used as the minimum energy structure in our simulations. Chains A and B from the PDB fibril structure are kept. Color codes are the same as in Fig. 2 and refer to the case of bottom side elongation. 

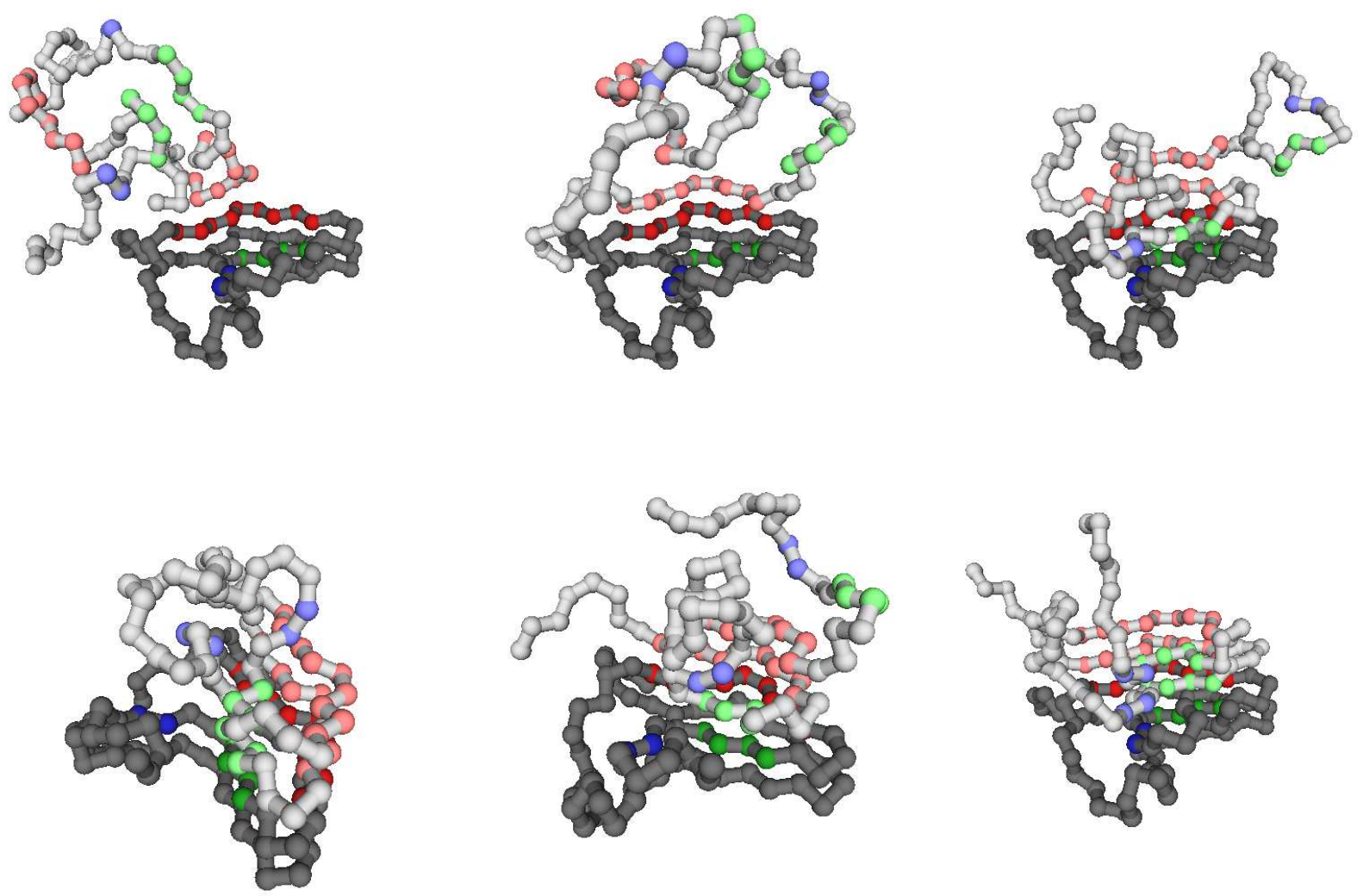

FIG. 4. Snapshot conformation from numerical simulations. Some snapshots representing conformations visited during the simulation of fibril elongation from the top side. Color codes are the same as in Fig. 2.
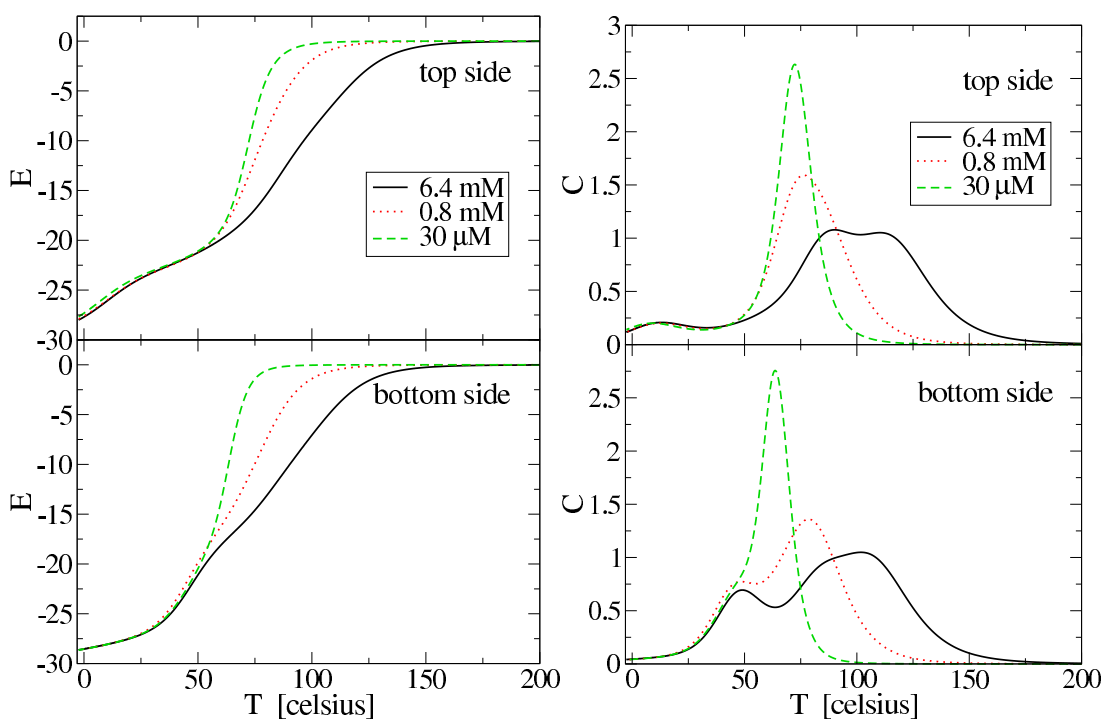

FIG. 5. Energy and heat capacity curves. Energy $E$ and heat capacity $C=d E / d T$ as a function of temperature $T$ in ${ }^{\circ} \mathrm{C}$, for both elongation sides and for the three different concentrations $c_{1}, c_{2}, c_{3}$ used in this work. Both $E$ and $C$ are plotted in simulation units so that 1 energy unit (minus 1 hydrogen bond) corresponds to $3.5 \mathrm{Kcal} \cdot \mathrm{mol}^{-1}$ (see Methods) while 1 heat capacity unit corresponds to the gas constant $R=1.99 \cdot 10^{-3} \mathrm{Kcal} \cdot \mathrm{mol}^{-1} \cdot \mathrm{K}^{-1}$. 

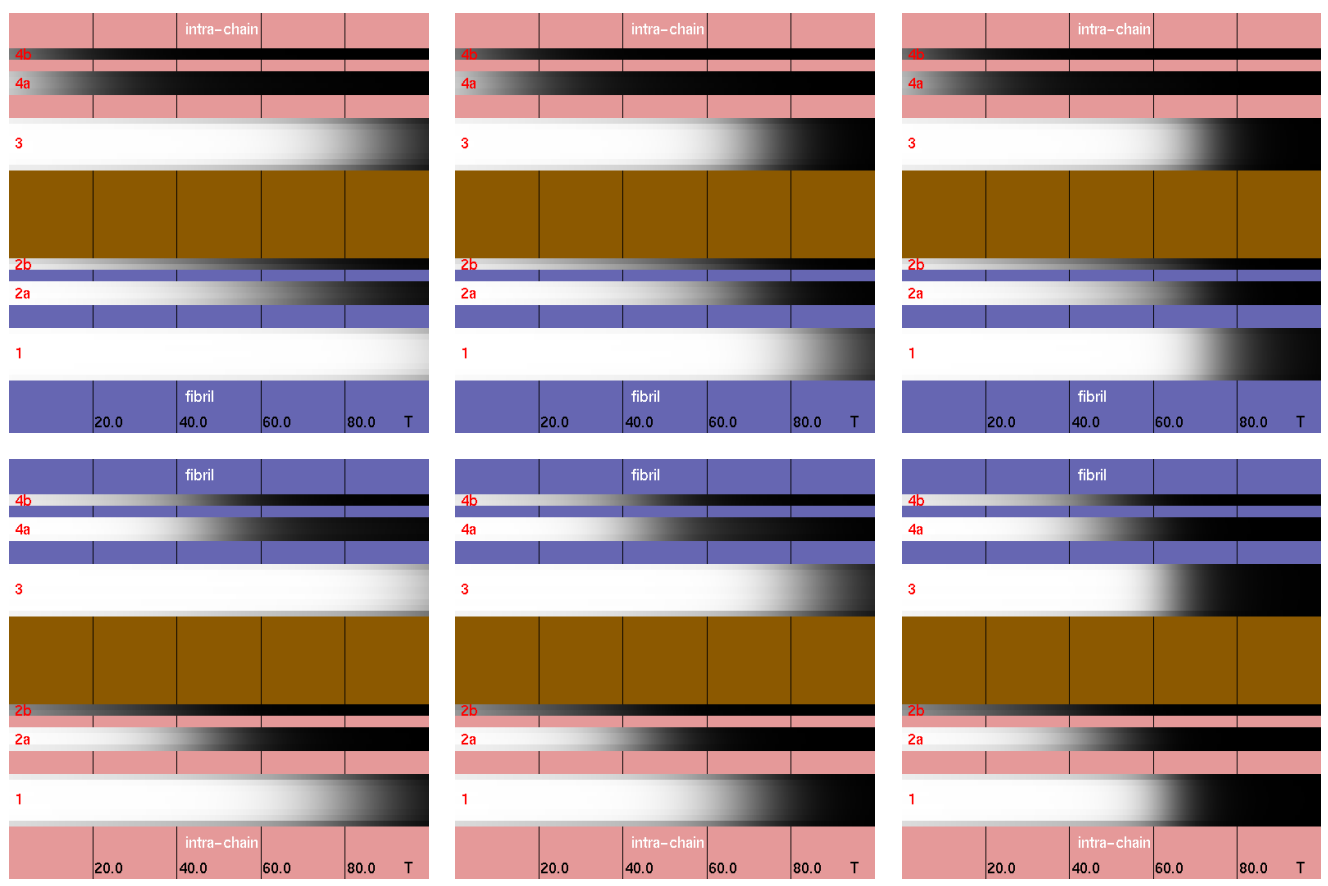

FIG. 6. Hydrogen bond formation probabilities. Plots of hydrogen bond formation probabilities in the mobile chain as a function of temperature $T$ in ${ }^{\circ} \mathrm{C}$ (white $=$ contact formed with probability 1 , black $=$ contact never formed), for top (upper row) and bottom (lower row) side elongation; columns refer to the three different concentrations $c_{1}$ (left), $c_{2}$ (middle), and $c_{3}$ (right) used in this work. We employ a representation in which hydrogen bonds formed between two residues are ascribed to the more 'external' residue of the two, according to the direction defined by fibril elongation. In all plots, this residue index is represented on the $y$-axis, from the $\mathrm{N}$-terminus to the $\mathrm{C}$-terminus. The lower wide black/white band corresponds to residues in the strand $\beta_{1}$ (9 hydrogen bonds), followed by residues in $\beta_{2 a}$ ( 4 hydrogen bonds) and $\beta_{2 b}$ ( 2 hydrogen bonds), and again residues in $\beta_{3}$ (9 hydrogen bonds), followed by residues in $\beta_{4 a}$ ( 4 hydrogen bonds) and in $\beta_{4 b}$ ( 2 hydrogen bonds). Strands are then divided into two larger groups, according to the nature of the associated hydrogen bonds, which may form intra-chain (pink band) or inter-chain (fibrillar blue band). The assignment of strands to the two groups depends on the elongation side. For instance, for top side elongation (upper row), strand $\beta_{1}$ of the mobile chain is on the 'internal' fibrillar side of the chain and the associated hydrogen bonds form inter-chain, with the strand $\beta_{3}$ of the fixed molecule. On the other hand, for bottom side elongation (lower row), strand $\beta_{1}$ of the mobile chain is on the 'external' exposed side of the molecule and the associated hydrogen bonds form intra-chain, with the strand $\beta_{3}$ of the same molecule. The brown band corresponds to the loop (246-260) separating the two groups of strands. 


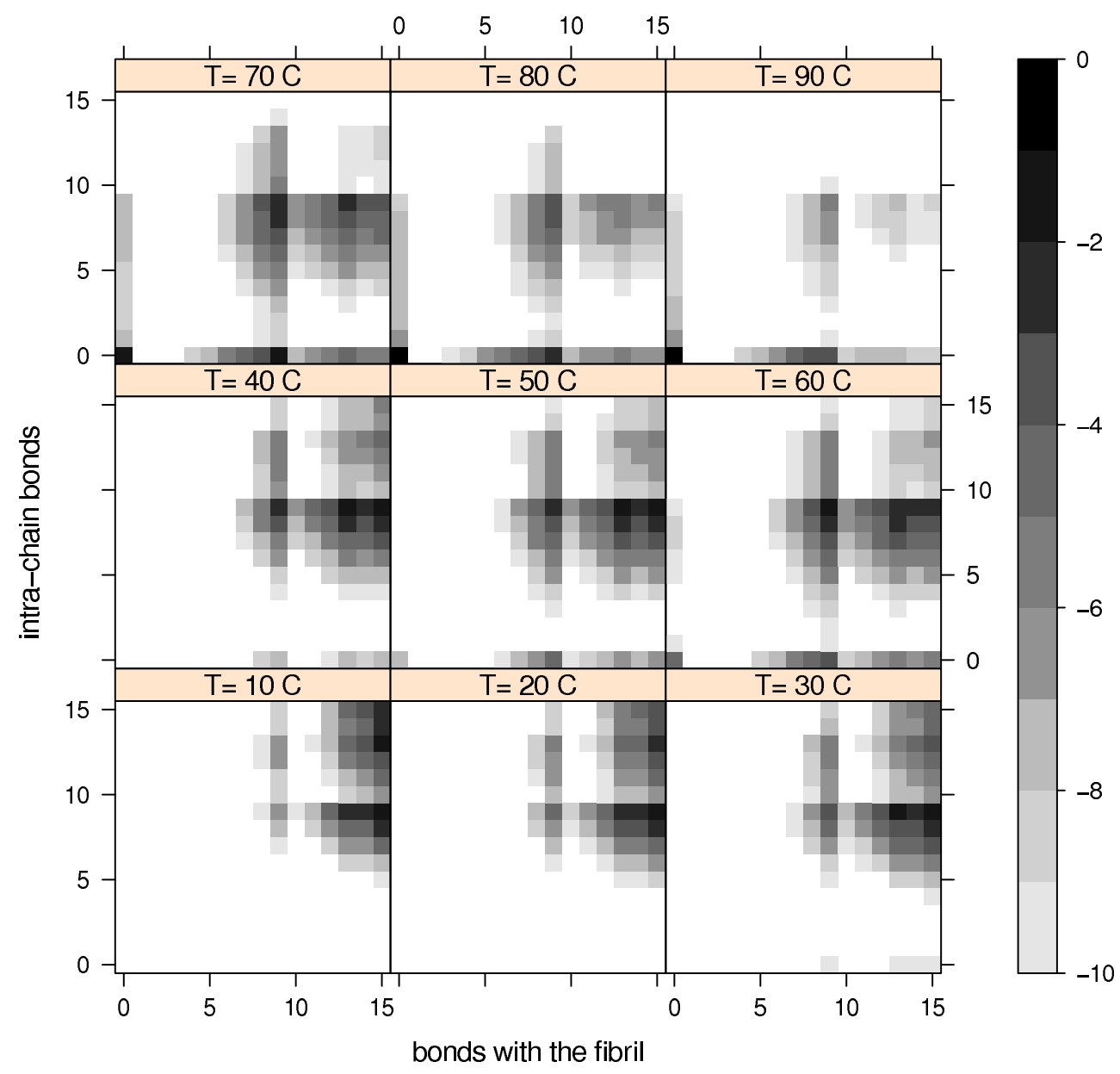

FIG. 7. Equilibrium occupation probabilities of macroscopic conformational states. Equilibrium occupation probability of conformational states, in logarithmic scale, shown for different temperatures $T$ in ${ }^{\circ} \mathrm{C}$, at concentration $c_{3}$ in the case of growth from the top side. Macroscopic states are defined according to the number of formed fibrillar and intra-chain hydrogen bonds, which are shown in the axes of each plot. The darker (lighter) the colour, the higher (smaller) the probability to occupy the corresponding macroscopic state. The numbers in logarithmic scale may be interpreted as the opposite of free energies in $R T$ units. 


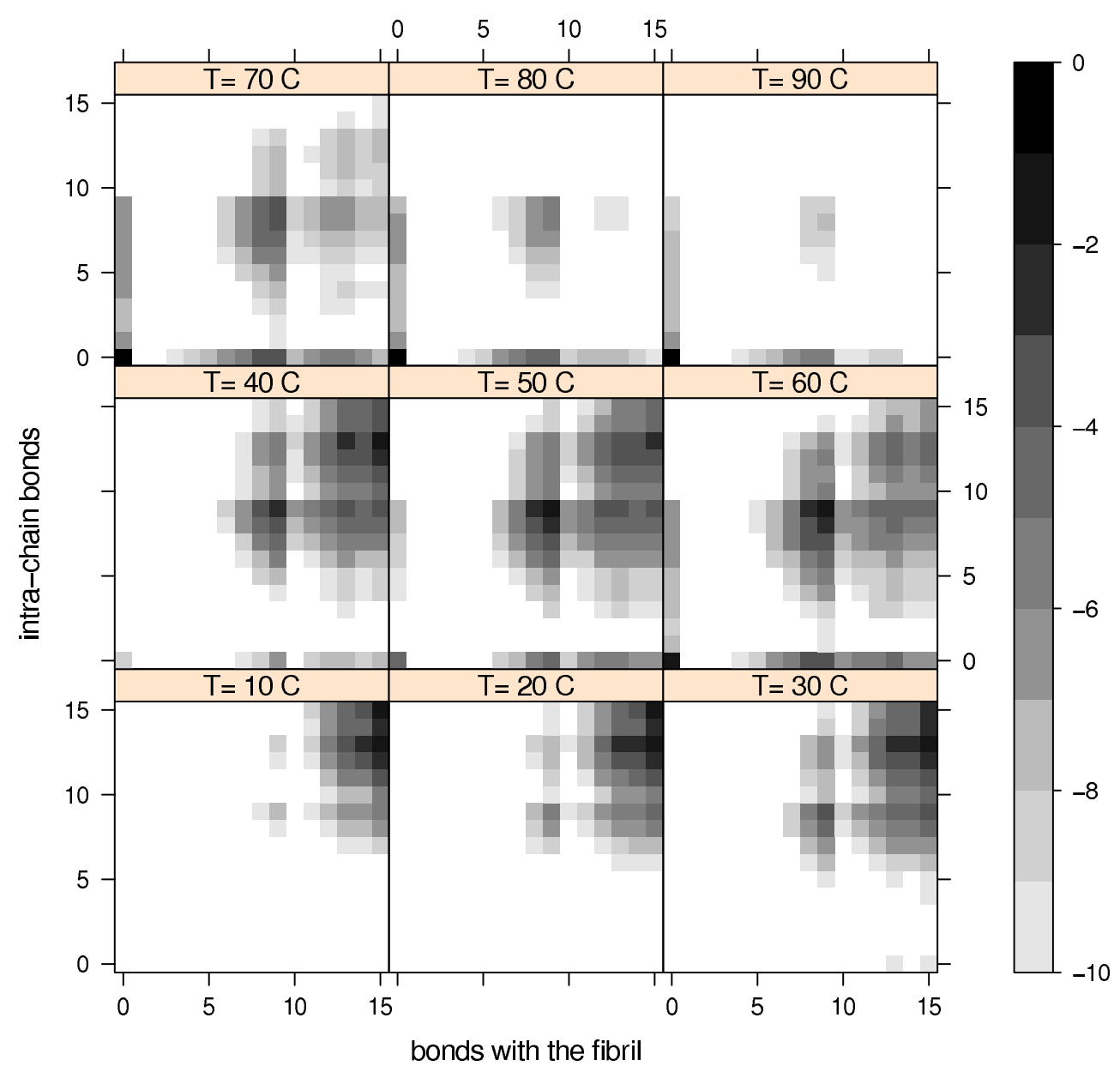

FIG. 8. Equilibrium occupation probabilities of macroscopic conformational states. As in Fig. 7, in the case of growth from the bottom side. 

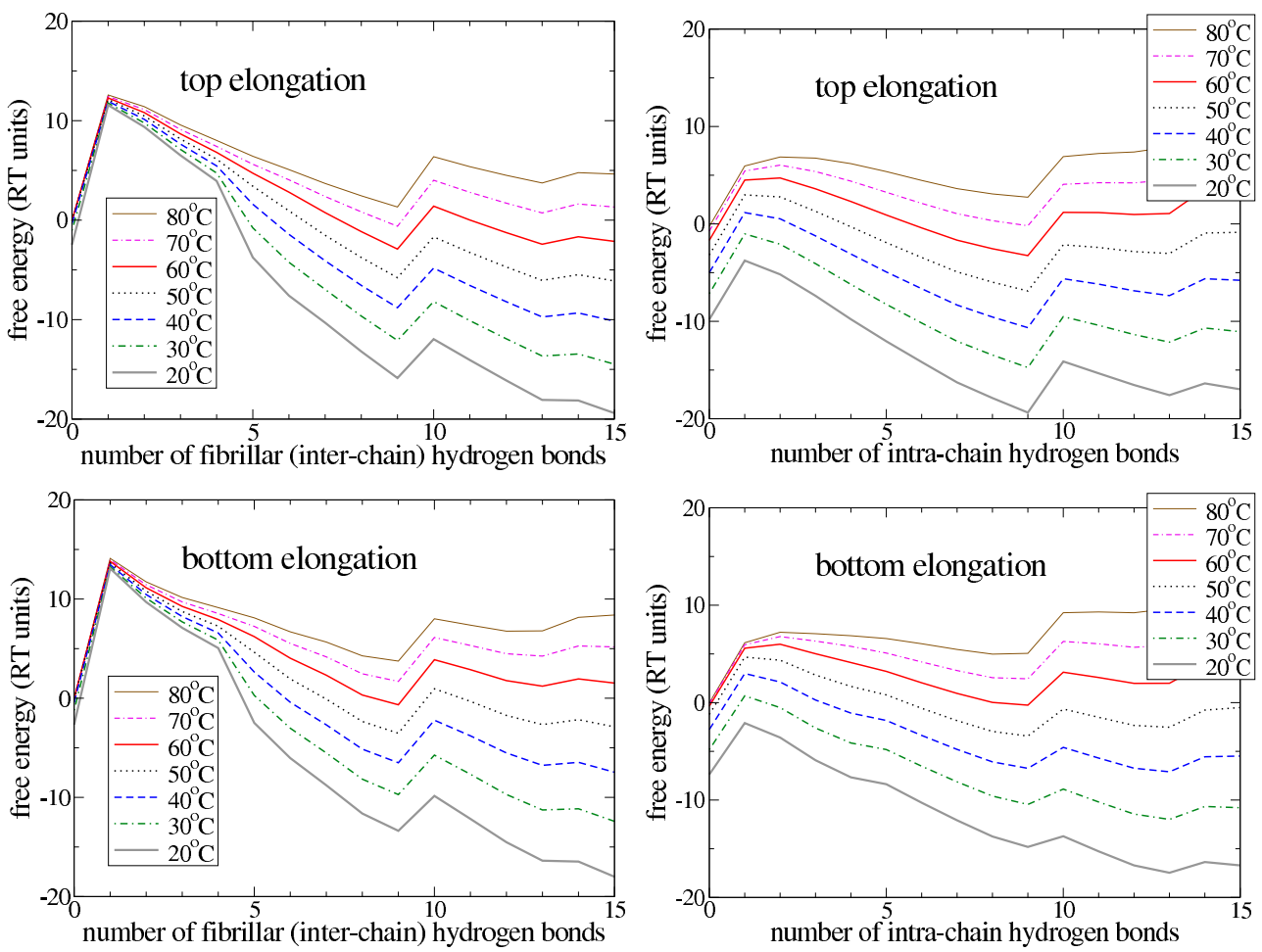

FIG. 9. Unidimensional energy profiles. Adimensonal free energy profiles (in $R T$ units), as a function of either the number of inter-chain fibrillar hydrogen bonds (left panels) or the number of intra-chain hydrogen bonds (right panels). Different profiles are shown for different temperatures in each panel, and both elongation modes are considered for the case of concentration $c_{3}$ : top elongation in the upper panels and bottom elongation in the lower panels.
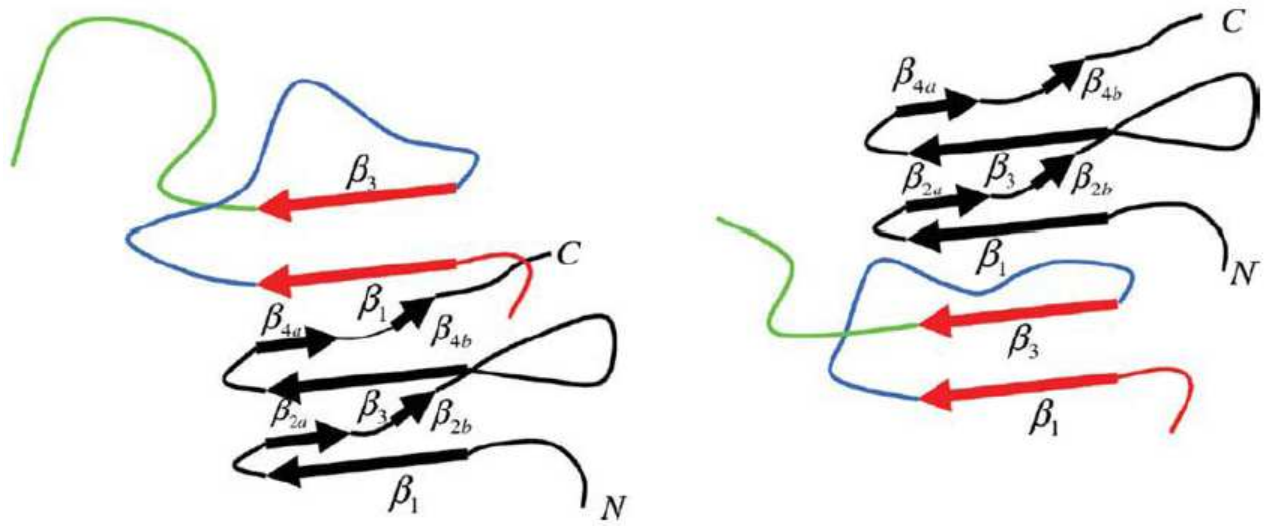

FIG. 10. Fibril growth polarity caused by topological properties. Cartoon representation of the elongating fibril after the initial docking of the attaching mobile chain (in colours) onto the fixed chain (in black - it represents the tip of the fibril) has just taken place and before the final locking stage. Only $\beta$-strands already formed are shown, in red for the mobile chain (the long strands). The remaining fluctuating portions of the mobile chain are shown in green (the 'tail') and in blue (the 'loop'). Right: top side elongation. Left: bottom side elongation. 